Now, if $m$ is odd, then $\left(m+1-2^{N-3}\right) / 2$ is an integer which may be taken as the value of $b$ since it satisfies the conditions (7). It is easily seen that the set (6), in which $b=\left(m+1-2^{N-3}\right) / 2$ and $c$ is determined by (8), is not the set (4). However, if $m$ is even and not equal to $2^{N-3}$, then $b=\left(m+2-2^{N-3}\right) / 2$ and $c$ determined by (8) are integers which satisfy (7) and yield a set (6) which is not the set (4). But if $m=2^{N-3}$, then $N \geqq 6$ and $b=2$ satisfies the conditions (7) on $b$ and yields a set (6) which is not the set (4).

An interesting choice of integers $b$ and $c$ is that given by $b=\left(m+1-2^{N-4}\right) / 2$ if $m$ is odd and less than or equal to $\left(2^{N-4}+2^{N-3}-1\right)$, but by $b=\left(m-2^{N-4}\right) / 2$ if $m$ is even and less than or equal to $\left(2^{N-4}+2^{N-3}\right)$. Then (6) require no rearrangement, and $c$ is respectively $b$ or $b+1$. The resulting integers (6) differ from (4) when $m \neq 2^{N-4}+2^{N-3}-1,2^{N-4}+2^{N-3}$.

NORTHWESTERN UNIVERSITY

\title{
HAUSDORFF METHODS OF SUMMATION WHICH INCLUDE ALL OF THE CESȦRO METHODS
}

\section{H. L. GARABEDIAN}

1. Introduction. The transformation ${ }^{1}$

$$
\sigma_{m}=\sum_{n=0}^{m} C_{m, n} \Delta^{m-n} c_{n} \cdot s_{n},
$$

where $c_{n}=\int_{0}^{1} u^{n} d \phi(u)$ and $\left\{s_{n}\right\}$ is a given sequence, defines a regular method of summation of the sequence $\left\{s_{n}\right\}$ provided that $\phi(u)$ is of bounded variation on the interval $0 \leqq u \leqq 1$, continuous at $u=0$, and

$$
\phi(u)=\left\{\begin{array}{l}
0 \quad \text { if } u=0, \\
1 \text { if } u=1, \\
\frac{1}{2}[\phi(u-0)+\phi(u+0)] \text { if } 0 \leqq u<1 .
\end{array}\right.
$$

If these conditions of regularity are fulfilled the sequence $\left\{c_{n}\right\}$ is said to be a regular moment sequence (briefly a regular sequence), the mass function $\phi(u)$ is said to be a regular mass function, and the method of summation involved is called a Hausdorff method of summation ([1] or [2]) and is designated by the symbol $[H, \phi(u)]$.

Presented to the Society, February 22, 1941; received by the editors April 8, 1941.

1 To define the symbolism used here we write $C_{m, n}=m(m-1) \cdots(m-n+1) / n !$, $C_{m, 0}=1 ; \Delta^{i} x_{j}=x_{j}-C_{i, 1} x_{j+1}+C_{i, 2} x_{j+2}+\cdots$. 
By properly specializing the mass function $\phi(u)$ it is possible to obtain various well known methods of summation. For example, the mass function $\phi(u)=1-(1-u)^{\alpha}, \alpha>0$, defines Cesàro summability $(C, \alpha)$. The regular sequence associated with this mass function is $c_{n}=1 / C_{\alpha+n, n},(n=0,1,2, \cdots)$.

It is the object of this note to exhibit new Hausdorff methods of summation which have the rare property of including Cesàro summability of all real and positive orders.

2. A mass function $\phi(u)$ independent of $\sigma$ for which $[H, \phi(u)]$ $\supset(C, \sigma), \sigma>0$. In order to exhibit a regular mass function $\phi(u)$ independent of $\sigma$ for which $[H, \phi(u)] \supset(C, \sigma), \sigma>0$, we write a theorem first stated by E. Hille and J. D. Tamarkin [3] which provides necessary and sufficient conditions in order that $[H, \phi(u)] \supset(C, n)$ $(n=1,2,3, \cdots)$. This theorem has recently been restated and proved in a paper by E. Hille, H. S. Wall, and this writer [4].

THEOREM 1. Necessary and sufficient conditions in order that $[H, \phi(u)] \supset(C, n), n \geqq 1$, are

(i) $\phi(u)$ is absolutely continuous and has absolutely continuous derivatives of order equal to or less than $n-1$ for $0<u \leqq 1$,

(ii) $\phi^{(n-1)}(u)$ has a finite right-hand derivative $\phi_{r}^{(n)}(u)$ for $0<u<1$, and a finite left-hand derivative $\phi_{l}^{(n)}(u)$ for $0<u \leqq 1$,

(iii) $\phi(u), u \phi^{\prime}(u), \cdots, u^{n-1} \phi^{(n-1)}(u), u^{n} \phi_{r}^{(n)}(u), u^{n} \phi_{l}^{(n)}(u)$ are of bounded variation on the interval $(0,1)$,

(iv) $1-\phi(u), \phi^{\prime}(u), \cdots, \phi^{(n-1)}(u)$ tend to zero as $u \rightarrow 1$,

(v) $\phi(u), u \phi^{\prime}(u), \cdots, u^{n-1} \phi^{(n-1)}(u), u^{n} \phi_{r}^{(n)}(u), u^{n} \phi_{l}^{(n)}(u)$ tend to zero as $u \rightarrow 0$.

The simplest regular mass function which we have been able to find which fulfills all of the five conditions of Theorem 1 for all values of $n \geqq 1$ is the function

$$
\phi_{a}(u)=1-\exp \{u /(u-1)\} .
$$

We observe that this function is monotone increasing on the interval $(0,1)$. If we modify $(2.1)$ by writing

$$
\phi(u)=\left[1-\exp \left\{-\alpha u /(1-u)^{\beta}\right\}\right]^{\gamma}, \quad \alpha, \beta, \gamma>0,
$$

we obtain a mass function which also fulfills the conditions of Theorem 1 for all $n \geqq 1$.

3. A theorem due to Hausdorff $[1$, p. 90]. If a regular mass function $\phi(u)$ is continuous on the interval $(0,1)$, and if furthermore we set $u=e^{-x}$, then the associated regular sequence takes the form 


$$
c_{n}=\int_{0}^{\infty} e^{-n x} \Phi(x) d x, \quad n=0,1,2, \cdots,
$$

where $\Phi(x)=e^{-x} \phi^{\prime}\left(e^{-x}\right)=u \phi^{\prime}(u)$. Hausdorff has proved the following theorem, involving the function $\Phi(x)$ instead of the mass function $\phi(u)$, which is closely related to Theorem 1 of the preceding section.

Theorem 2. If the functions. $\Phi(x), \Phi^{\prime}(x), \Phi^{\prime \prime}(x), \cdots$ exist for $x>0$, if they are absolutely integrable on the interval $(0, \infty)$, and if they tend to zero as $x \rightarrow 0$, then the sequence (3.1) defines a regular method of summation which includes all of the Cesàro methods.

The hypotheses of this theorem imply that all of the functions involved tend to zero as $x \rightarrow+\infty$. With this observation it is readily verified that the mass function

$$
\phi(u)=\int_{0}^{u} \frac{1}{t} \Phi\left(\log \frac{1}{t}\right) d t,
$$

when $\Phi(x)$ satisfies the conditions of Theorem 2, fulfills the conditions of Theorem 1 for all $n \geqq 1$.

Examples due to Hausdorff of functions which fulfill the conditions of Theorem 2 are:

$$
\begin{array}{lr}
\Phi(x)=A \exp \{-a x-b / x\} x^{\lambda-1}, & a, b>0 ; \lambda \text { arbitrary, } \\
\Phi(x)=B e^{-(b / x)} x^{\lambda-1}, & b>0 ; \lambda<0,
\end{array}
$$

where $A$ and $B$ are normalizing factors which make $c_{0}=1$. If in particular we have $\Phi(x)=e^{-1 / 4 x} x^{-3 / 2} /\left(2 \pi^{1 / 2}\right)$, the associated moment sequence is $c_{n}=e^{-n^{1 / 2}},(n=0,1,2, \cdots)$, and the associated regular mass function is

$$
\phi_{b}(u)=\frac{1}{2 \pi^{1 / 2}} \int_{0}^{u} \frac{1}{t} \exp \{1 / 4 \log t\}\left(\log \frac{1}{t}\right)^{-3 / 2} d t .
$$

Like the function $\phi_{a}(u)$ of $(2.1)$, the function $\phi_{b}(u)$ is a monotone increasing function on the interval $(0,1)$.

In comparing the mass functions $\phi_{a}(u)$ and $\phi_{b}(u)$ we notice that $\phi_{a}(u)$ is much simpler in form than $\phi_{b}(u)$. On the other hand the regular sequence associated with $\phi_{b}(u)$ has a simple form, while the regular sequence associated with $\phi_{a}(u)$ cannot be computed in elementary terms.

4. The difference matrix. In a recent paper [5] H. S. Wall and this writer have discussed properties of the difference matrix 
$\Delta=\left(\Delta^{m} c_{n}\right)$, where $\left\{c_{n}\right\}$ is a regular sequence. In particular it was observed that

$$
\Delta^{m} c_{n}=\int_{0}^{1} u^{n}(1-u)^{m} d \phi(u)
$$

and hence that the sequence $\left(\Delta^{m} c_{0}, \Delta^{m} c_{1}, \Delta^{m} c_{2}, \cdots\right)$ is an essentially regular sequence, ${ }^{2}$ the associated mass function being $\int_{0}^{u}(1-t)^{m} d \phi(t)$. We observe now for the first time that $m$ need not be a positive integer, the integral in (4.1) affording a definition for the fractional differences involved here.

If, for example, the mass functions $\phi_{a}(u)$ and $\phi_{b}(u)$ are inserted in the difference matrix new methods of summation are generated which have the property of including all of the Cesàro methods. The method of summation thus generated by the mass function $\phi_{a}(u)$ has the mass function

$$
\phi_{c}(u)=A \int_{0}^{u}(1-t)^{c-2} \exp \{t /(t-1)\} d t, \quad c \geqq 0,
$$

where $A$ is a normalizing factor which insures that $\phi_{c}(1)=1$. On the basis of known results arising from inclusion problems in the difference matrix [5] we make the conjecture that summability $\left[H, \phi_{c}(u)\right]$ increases in efficiency with increasing $c$.

\section{REFERENCES}

1. F. Hausdorff, Summationsmethoden und Momentfolgen, I and II, Mathematische Zeitschrift, vol. 9 (1921), pp. 74-109, 280-299.

2. H. L. Garabedian, Hausdorff matrices, American Mathematical Monthly, vol. 46 (1939), pp. 390-410.

3. E. Hille and J. D. Tamarkin, Questions of relative inclusion in the domain of Hausdorff means, Proceedings of the National Academy of Sciences, vol. 19 (1933), pp. 573-577.

4. H. L. Garabedian, E. Hille, and H. S. Wall, Formulations of the Hausdorff in clusion problem, Duke Mathematical Journal, vol. 8 (1941), pp. 193-213.

5. H. L. Garabedian and H. S. Wall, Hausdorff methods of summation and continued fractions, Transactions of this Society, vol. 48 (1940), pp. 185-207.

\section{NORTHWESTERN UNIVERSITY}

2 An essentially regular sequence may be normalized or made regular by dividing every term of the sequence by its first term. 